\title{
Creatinine in urine - a method comparison
}

Authors:

Marc Luginbühl ${ }^{1}$, Wolfgang Weinmann ${ }^{1}$

Affiliations:

${ }^{1}$ Institute of Forensic Medicine, University of Bern, Switzerland

Addresses:

${ }^{1}$ Institute of Forensic Medicine, University of Bern

Bühlstrasse 20

3012 Bern

Switzerland

Corresponding author:

Marc Luginbühl

Institute of Forensic Medicine, University of Bern

Bühlstrasse 20

3012 Bern

Switzerland

Email: marc.luginbuehl@irm.unibe.ch

\section{Creatinine in urine - a method comparison}

Marc Luginbühl*, Wolfgang Weinmann

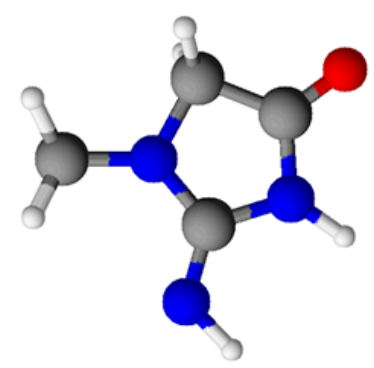

Three different methods for the determination of creatinine concentrations in urine are carefully investigated. LC-MS/MS proved to be advantageous compared to a spectrophotometric or point-of-care testing based analysis method. 


\begin{abstract}
Drug screening in urine is widely applied in forensic toxicology. Contrary to blood analysis, excessive or reduced fluid intake can substantially alter the concentration of substances in urine. As a standard to detect urinary dilution, creatinine concentrations are analyzed. A sample with a concentration below $20 \mathrm{mg} / \mathrm{dL}$ is generally defined as too diluted to provide a valid result in abstinence control samples. This work investigates the potential of three different methods for the determination of creatinine concentrations in urine samples: A ZIC-HILIC based LC-MS/MS method, a spectrophotometric method on an AU 480 chemistry system, and a portable, chemical reaction based, point-of-care testing device was compared by measuring 200 urine samples. When comparing the two laboratory methods, LC-MS/MS and spectrophotometry, a mean difference of $3.7 \pm 14 \mathrm{mg} / \mathrm{dL}$ was found, indicating that the spectrophotometric method is slightly overestimating the creatinine concentration. When comparing the LC-MS/MS method with the point-of-care testing device, a mean concentration difference within the calibration range for POCT ( $>20 \mathrm{mg} / \mathrm{dL}$ (excluding 16 samples) and $<500 \mathrm{mg} / \mathrm{dL}$ (excluding 4 samples)) of $21 \pm 37 \mathrm{mg} / \mathrm{dL}$ was found, indicating that the point-of-care testing device overestimates the measured creatinine concentration. A point-of-care testing device as used during this study can provide valuable information for on-site analysis. However, reported concentrations above $500 \mathrm{mg} / \mathrm{dL}$ should be further evaluated e.g. by dilution of the sample.
\end{abstract}

Keywords. - creatinine, urine analysis, drug testing, point-of-care testing, urine manipulation 


\section{Introduction}

As excessive or reduced fluid intake can substantially alter the concentration of substances in urine, the necessity of investigating the urine's dilution when measuring drugs of abuse (DOA) or performing ethyl glucuronide testing is widely known $(1,2)$. This is usually done by measuring creatinine, the internal anhydride of creatine (3). Based on a normal range of creatinine excretion from 14 to $26 \mathrm{mg} / \mathrm{kg}$ per day for men and 11 to $20 \mathrm{mg} / \mathrm{kg}$ per day for women, the urine's creatinine concentration can vary massively, because the individual urinary creatinine output correlates better with muscle mass than with body weight (2). An obese person is thereby expected to have a lower urinary creatinine excretion than an athletic individual with the same or even lower body mass (3). Nevertheless, the measured urinary creatinine concentration is frequently used to provide information about the dilution, independent of the individual body composition of the donor: According to the European guidelines for workplace drug testing in urine, samples with a creatinine concentration below $22.6 \mathrm{mg} / \mathrm{dL}$ should be considered as dilute (4). Dilution of the urine may lead to an underestimation or non-detectability of the drug concentration (2).

In this study, we provide a comparison between two laboratory methods for the urine's creatinine analysis: liquid chromatography - mass spectrometry and spectrophotometry. In parallel, a pointof-care testing (POCT) device based on the oxidation of an indicator dye by a creatinine/copper complex and a hydroperoxide is evaluated by measuring the same urine samples as with the two laboratory methods. Knowledge about the reliability of such a portable device may help the investigator on-site, e.g. during roadside testing, to decide if an additional blood test, to detect drugs of abuse, is advisable. We refrained from testing the urine samples with an enzymatic assay using the Trinder Reaction, as various substances such as N-acetylcysteine (NAC), calcium dobesilate, acetaminophen and the metabolite $\mathrm{N}$-acetyl-p-benzoquinone, metamizole and the metabolites 4aminoantipyridine (4-AAP) and 4-methylaminoantipyrine (4-MAP) cause falsified results for

Trinder based assays (5-7). Acetaminophen and metamizole are often present in the studied population.

\section{Materials and methods}

\section{Reagents}

Creatinine and creatinine- $d_{3}$ were obtained from Sigma-Aldrich (St Louis, USA). Ammonium acetate fractopur ${ }^{\circledR}$ was ordered from Merck (Darmstadt, Germany). Acetonitrile (p.a.) was bought from Acros Organics (Geel, Belgium). Water was produced with a Milli-Q water system from Millipore (Billerica, USA). Creatinine reagent (Creatinine urine kit OSR 6178) for spectrophotometric calibration was obtained from Beckman Coulter (Nyon, Switzerland). A liquid assayed urine control for spectrometry (MAS Urichem Trak) was obtained from Thermo Fischer Scientific (Reinach, Switzerland). The P.I.A. ${ }^{2}$ POCT device and creatinine select plate tests were provided by Protzek (Lörrach, Germany). 


\section{Study design}

89 urine samples used in this study were obtained from eight healthy volunteers participating in a drinking study (8). Further 111 samples reached the institute of forensic medicine for routine analysis (driving under the influence of drugs) and were analyzed for their creatinine concentration. The determination of the creatinine concentration by spectrophotometry represents a standard procedure which is generally performed for all urine samples sent to our laboratory. All samples were anonymized prior to analysis by using a specific sample identification code. In total 200 urine samples were analyzed for their creatinine concentration with three different methods: LC-MS/MS, spectrophotometry, and a chemical reaction based POCT device.

\section{Determination of creatinine by LC-MS/MS}

Sample preparation, analysis, and validation has recently been described in detail (8). Briefly, 50 $\mu \mathrm{L}$ of urine and $150 \mu \mathrm{L}$ acetonitrile containing the internal standard (creatinine- $d_{3}$ ) were shaken for several minutes. After centrifugation, the supernatant was taken and evaporated to dryness. Prior to instrumental analysis, the sample was reconstituted in $400 \mu \mathrm{L}$ of reconstitution buffer. Chromatographic separation was performed with a SeQuant ${ }^{\circledR}$ ZIC $^{\circledR}$-HILIC $3.5 \mu \mathrm{m}, 100 \AA$ A, $150 \mathrm{x}$ $2.1 \mathrm{~mm}$ PEEK coated HPLC column (Merck, Darmstadt, Germany), heated at $30{ }^{\circ} \mathrm{C}$, with a flow rate of $0.3 \mathrm{~mL} / \mathrm{min}$. Mobile phase A consisted of a $5 \mathrm{mM}$ ammonium acetate buffer at $\mathrm{pH} 5.72$ with $0.1 \%$ formic acid, mobile phase B consisted of acetonitrile with $0.1 \%$ formic acid. The following 14 min gradient was used: 0 to $1 \mathrm{~min}, 95 \%$ B; 1 to $4.5 \mathrm{~min}, 95 \%$ to $20 \%$ B linear; 4.5 to 9 min, 20\% B; 9 to $10 \mathrm{~min} 20 \%$ to $95 \%$ B linear; 10 to $14 \mathrm{~min}$, 95\% B. Mass spectrometric detection was performed on a Sciex 5500 QTrap system, operated in electrospray negative SRM mode (monitored transitions for crea: quantifier: $112 \rightarrow 68$, qualifier: $112 \rightarrow 41$, internal standard signal crea- $d_{3}$ : $115 \rightarrow 42)$. Creatinine eluted from the column with a retention time of 5 minutes. Calibration was performed with a five point calibration prepared in solvent (2, 10, 30, 60, and $250 \mathrm{mg} / \mathrm{dL})$, using a linear calibration model with weighting $1 / \mathrm{x}^{2}$. The correlation coefficient $\left(\mathrm{R}^{2}\right)$ from least square regression were at least 0.9990 . Four quality control samples (2, 10, 20, and $130 \mathrm{mg} / \mathrm{dL}$ ) were used in each run. Samples with concentrations above the highest calibrator were reinjected after dilution. Mean intra-assay accuracy by measuring six samples of each quality control was 98.8-101.7\%, and mean imprecision was 0.7-2.4\%. Mean inter-assay accuracy from three series $(n=3)$ with six samples of each quality control in each series was 98.9-101.4\%, and mean imprecision was 0.62.5\%. Furthermore, extraction efficiency was determined to be $55 \pm 13 \%$, the recovery was $53 \pm$ $11 \%$, and the matrix effects were $96 \pm 12 \%$. The extracted quality control samples proved to be stable after storage for $72 \mathrm{~h}$ in the autosampler, after three freeze thaw cycles, and after storage for 7 days at $4{ }^{\circ} \mathrm{C}$.

\section{Determination of creatinine by spectrophotometry}

Spectrophotometric determination of creatinine was performed on a Beckman Coulter AU 480 (Nyon, Switzerland). The colorimetric method is based on a kinetic test (Jaffe-reaction), whereby creatinine forms a complex with picric acid, as the deviation in absorption at 520/800 nm is 
proportional to the creatinine concentration within the sample $(9,10)$. The determination is based on a one-point calibration at $153 \mathrm{mg} / \mathrm{dL}$, which is forced through zero. Commercially available quality control urine with a creatinine concentration of $57 \mathrm{mg} / \mathrm{dL}$ (acceptable deviation $<20 \%$ ) was included. According to manufacturer specification, the creatinine test shows linearity between 1 to $400 \mathrm{mg} / \mathrm{dL}$ (10). A seven point linearity test (0, 10, 20, 50, 100, 200, 300, $400 \mathrm{mg} / \mathrm{dL})$ in our laboratory revealed a correlation coefficient $\left(\mathrm{R}^{2}\right)$ from least square regression of 0.9998 . Based on repetitive measurements of the quality control sample during two months $(n=17)$, mean imprecision was found to be $6.1 \%$.

\section{Determination of creatinine by POCT}

POCT was performed on lateral flow, creatinine select plate tests with an automatic P.I.A ${ }^{2}$ readout system from Protzek (Lörrach, Germany), see figure 1. The test is a chemical reaction based device, using copper ions for complexation of the creatinine and a hydroperoxide for the oxidation of an indicator dye for color development of the test field. The calibration model for the determination of the creatinine concentration is based on a saturation curve by comparing the peak-volume (brightness of the reaction pad mounted on a lateral flow device) with the spiked concentration, which is established by measuring eight samples with different concentrations $(0,20,40,75,125$, 200, 300, and $500 \mathrm{mg} / \mathrm{dL}$ ) (figure 2). Accuracy measurements by comparing 24 human urine samples with spectrophotometric measurements revealed a mean accuracy of $112.5 \%$ and a correlation coefficient $\left(\mathrm{R}^{2}\right)$ of 0.9453 . Precision measurements during five days with a low (50 $\mathrm{mg} / \mathrm{dL})$ and a high (100 mg/dL) creatinine concentration resulted in a mean imprecision of $10 \%$ and 5.7\%, respectively. To investigate potential interferences, various substances such as glucose (100 mg/dL), uric acid (100 mg/dL), urea (3000 mg/dL), sodium chloride (1000 mg/dL), and proteins (BSA) $(1000 \mathrm{mg} / \mathrm{dL})$ were spiked to a $100 \mathrm{mg} / \mathrm{dL}$ creatinine stock solution in triplicate, whereby no influence on the test result was observed (mean imprecision <8\%). For sample acquisition the following steps were performed: The provided buffer solution (400 $\mu$ l, provided with the test cartridge) was mixed with $100 \mu \mathrm{L}$ of urine, by using a Gilson Pipetman ${ }^{\circledR} 200 \mathrm{M}$ (systematic error $\pm 1.00 \mu \mathrm{L}$, random error $\leq 0.26 \mu \mathrm{L}$ ). The mixture was shaken for ten seconds at $1,500 \mathrm{rpm}$ using a microtube shaker. Afterwards, $100 \mu \mathrm{L}$ of the mixture were pipetted on the plate test and measured by automatic photometric readout on the P.I.A. ${ }^{2}$ system after three minutes. The result was provided in $\mathrm{mg} / \mathrm{dL}$ of creatinine with a measurement window of 20 to $500 \mathrm{mg} / \mathrm{dL}$. Alternatively, the device can be used in a direct interpretation mode, as shown in table 1 (11). Each sample was analyzed by a single measurement.

\section{Results and discussion}

\section{LC-MS/MS vs. spectrophotometry}

When comparing the two laboratory methods, a Bland-Altman plot revealed a mean difference of $-3.7 \pm 14.3 \mathrm{mg} / \mathrm{dL}$, indicating that the spectrophotometric method is overestimating the creatinine concentration, see figure 3 . A one sample t-test reveals a $\mathrm{P}$ value of $1.61 \mathrm{E}-4$, showing that the 
measured difference between the two methods is highly significant, which indicates the presence of a fixed bias. Linear regression analysis reveals a slope with $y=0.9732 x+6.85$ and a $R^{2}$ value of 0.9683 , see figure 4 . This results in an average systematic error of $3.7 \mathrm{mg} / \mathrm{dL}(\mathrm{y}=0.9732 \overline{\mathrm{x}}+6.85$, whereby $\overline{\mathrm{x}}$ for the LC-MS/MS measurement equals $116.90 \mathrm{mg} / \mathrm{dL}$ ) for the spectrophotometric measurement, which is in agreement with the previously mentioned mean difference of $3.7 \pm 14.3$ $\mathrm{mg} / \mathrm{dL}$ from the Bland-Altman plot. When comparing the LC-MS/MS results close to the cutoff concentration (30 mg/dL) for diluted urine, from 0 to $100 \mathrm{mg} / \mathrm{dL}$ (with respect to the LC-MS/MS result), linear regression analysis results in a slope of $\mathrm{y}=1.1484 \mathrm{x}-2.80$ and a $\mathrm{R}^{2}$ value of 0.9435 , see figure 5 . This indicates that the correlation coefficient between the two methods decreases as the observed relative concentration differences increase, when only low creatinine concentrations are investigated. A Bland-Altman plot revealed a mean difference of $-5.0 \pm 9.0 \mathrm{mg} / \mathrm{dL}$ for samples from 0 to $100 \mathrm{mg} / \mathrm{dL}$. Reanalysis of ten randomly selected urine samples resulted in a mean difference of $-10 \pm 17 \mathrm{mg} / \mathrm{dL}$ for the LC-MS/MS analysis compared to $-29 \pm 13 \mathrm{mg} / \mathrm{dL}$ for the spectrophotometric analysis. Spiking of these ten urine samples with an additional $100 \mathrm{mg} / \mathrm{dL}$ of creatinine in order to investigate matrix effects resulted with an additional creatinine concentration of $105 \pm 9 \mathrm{mg} / \mathrm{dL}$ for the LC-MS/MS measurement and $104 \pm 11 \mathrm{mg} / \mathrm{dL}$ for the spectrophotometric measurement.

When analyzing 20 urine samples in order to compare LC-MS/MS and the spectrophotometric method, the group of Derezinski et al. found a high correlation with a $\mathrm{R}^{2}$ value of 0.9844 between both methods. In $80 \%$ of the tested samples, the Jaffe method demonstrated an overestimation, by $4.6 \%$ on average (12). Askenazi et al. found significantly lower urinary creatinine concentrations if samples were measured by the spectrophotometric Jaffe method compared to LC-MS/MS. The observed differences were however less than any clinically meaningful difference (13). A potential reason for this difference could be the preparation of calibration samples. It has to be mentioned, that a LC-MS/MS based method has less interfering substances and shows a higher specificity when it comes to creatinine analysis. This is mainly owed to the chromatographic separation of substances. On the other hand, numerous interferences such as acetone, cefazolin, glucose, bilirubin and lipids were found to influence the outcome of a spectrophotometric measurement (12).

\section{LC-MS/MS vs. POCT}

When comparing the LC-MS/MS method with the POCT device, a Bland-Altman plot within the calibration range for POCT (>20 mg/dL (excluding 16 samples) and <500 mg/dL (excluding 4)) revealed a mean difference of $-20.7 \pm 36.7 \mathrm{mg} / \mathrm{dL}$ creatinine, indicating that the POCT device is overestimating the creatinine concentration, see figure 6 . With outlier elimination $(n=20,<20$ and $>500$ ), one sample t-test reveals a $\mathrm{P}$ value of $1.25 \mathrm{E}-12$, showing that the measured difference between the two methods is significant, thus having a fixed bias. Linear regression analysis reveals a slope with $\mathrm{y}=1.1513 \mathrm{x}+1.80$ and an $\mathrm{R}^{2}$ value of 0.8631 , see figure 7 . This reveals an average systematic error of $20.7 \mathrm{mg} / \mathrm{dL}(\mathrm{y}=1.1513 \overline{\mathrm{x}}+1.80$, whereby $\overline{\mathrm{x}}$ for the LC-MS/MS measurement 
equals $124.61 \mathrm{mg} / \mathrm{dL}$ ) for the POCT device, which is in agreement with the previously mentioned mean difference of $20.7 \pm 36.7 \mathrm{mg} / \mathrm{dL}$ from the Bland-Altman plot. Based on the observed data, it is recommended to repeat measurements at the upper range of the scale ( $>500 \mathrm{mg} / \mathrm{dL}, \mathrm{n}=4)$, as they proved to be invalid test results. Samples with a creatinine concentration $<20 \mathrm{mg} / \mathrm{dL}$ (measured by POCT), accounting for dilute urine, were reliable in 15 out of 16 cases. In one case the POCT device found a creatinine concentration below $20 \mathrm{mg} / \mathrm{dL}$, whereas the LC-MS/MS determination revealed a concentration of $41 \mathrm{mg} / \mathrm{dL}$. Based on literature research, a direct comparison with another portable device for the determination of creatinine in urine is not possible. The currently available POCT devices for creatinine analysis such as the i-STAT (Abbott Point-of-Care, UK) and the Reflotron Plus (Roche Diagnostics, Switzerland) are intended to measure creatinine in whole blood, serum or plasma (14). Based on a review article by Mark DS Shephard, currently available POCT devices for creatinine measurements in blood can be divided into two types: blood gas analyzers such as the i-STAT and "non blood gas" analyzers such as the Reflotron Plus (15). 


\section{Conclusion}

The observed differences between the three methods can be seen as significant (based on the one sample t-test). As expected, the two laboratory methods LC-MS/MS and spectrophotometry are reliable and provide creatinine concentrations close to each other. LC-MS/MS measurements showed the highest reproducibility. Depending on the liquid chromatography used, creatinine determination can be implemented, within-run, in an already existing LC-MS/MS method for drugs-of-abuse testing. When comparing the POCT device with the LC-MS/MS method, differences in creatinine concentration are more distinct. However, with respect to diluted urine, 15 out of 16 samples with a creatinine concentration below $20 \mathrm{mg} / \mathrm{mL}$ (measured by POCT) were identified correctly. It was therefore found that the POCT device may provide a useful tool for the on-site testing of creatinine concentrations. Especially the fact that the device interprets the test outcome automatically was appreciated. However, particular attention has to be paid when creatinine values above $500 \mathrm{mg} / \mathrm{dL}$ are reported (4 out of 200 samples, which represents 2\%). In this case, a repetition of the measurement is recommended.

\section{Acknowledgements}

We would like to thank the team of the Forensic Toxicology and Chemistry Laboratory of the Institute of Forensic Medicine Bern for running samples with the AU 480 analyzer. Furthermore, we would like to extend our gratitude to the Protzek $\mathrm{GmbH}$ for providing us the P.I.A. ${ }^{2}$ system and test plates.

\section{Conflict of interest}

The authors declare that they have no conflict of interest

\section{Compliance with ethical standards}

The participants in the drinking study gave informed consent for the analysis of creatinine in the collected samples. Creatinine analysis of samples reaching the institute for routine analysis is a standard procedure. All samples were anonymized prior to analysis by using a specific sample identification code. 


\section{References}

1. Cone EJ, Caplan YH, Moser F, Robert T, Shelby MK, Black DL. Normalization of urinary drug concentrations with specific gravity and creatinine. Journal of analytical toxicology. 2009 Jan-Feb;33(1):17. PubMed PMID: 19161663. Epub 2009/01/24. eng.

2. Lafolie P, Beck O, Blennow G, Boreus L, Borg S, Elwin CE, et al. Importance of creatinine analyses of urine when screening for abused drugs. Clin Chem. 1991 Nov;37(11):1927-31. PubMed PMID: 1934467. Epub 1991/11/01. eng.

3. Narayanan S, Appleton HD. Creatinine: a review. Clinical Chemistry. 1980;26(8):1119-26.

4. European Guidelines for Workplace Drug Testing in Urine http://www.ewdts.org/ewdtsguidelines.html2015 [cited 2016 26.09.2016].

5. Genzen JR, Hunsaker JJ, Nelson LS, Faine BA, Krasowski MD. N-acetylcysteine interference of Trinder-based assays. Clinical biochemistry. 2016 Jan;49(1-2):100-4. PubMed PMID: 26500003. Epub 2015/10/27. Eng.

6. Guo X, Hou La, Cheng X, Zhang T, Yu S, Fang H, et al. Strong Negative Interference by Calcium Dobesilate in Sarcosine Oxidase Assays for Serum Creatinine Involving the Trinder Reaction. Medicine. 2015 06/12;94(23):e905. PubMed PMID: PMC4616468.

$7 . \quad$ Roche. Drug Interference in tests based on Trinder Reaction. Field Safety Notice FSN-RPD-2014008 in RPF/ClinChem fully automated. 2015.

8. Luginbühl M, Rutjens S, König S, Furrer J, Weinmann W. N-Acetyltaurine as a novel urinary ethanol marker in a drinking study. Analytical and Bioanalytical Chemistry. 2016:1-8.

9. Greenwald I. The Chemistry of Jaffe's Reaction for Creatinine II. The Effect of Substitution in the Creatinine Molecule and possible Formula for the red Tautomer 1. Journal of the American Chemical Society. 1925 1925/05/01;47(5):1443-8.

10. BeckmanCoulter. Creatinine OSR6178/OSR6578 manual. 2011.

11. Protzek. P.I.A. Tests, PIA2 CREA, user manual. 2014 17.04.2014:2.

12. Derezinski P, Klupczynska A, Sawicki W, Kokot ZJ. CREATININE DETERMINATION IN URINE BY LIQUID CHROMATOGRAPHY-ELECTROSPRAY IONIZATION-TANDEM MASS SPECTROMETRY METHOD. Acta poloniae pharmaceutica. 2016 Mar-Apr;73(2):303-13. PubMed PMID: 27180423. Epub 2016/05/18. Eng. 13. Askenazi DJ, Moore JF, Fineberg N, Koralkar R, Clevenger S, Sharer JD. Comparison of methods, storage conditions, and time to analysis of serum and urine creatinine measured from microsamples by liquid chromatography mass spectrometery (LC/MS) vs. Jaffe. Journal of clinical laboratory analysis. 2014 Sep;28(5):405-8. PubMed PMID: 24652788. Epub 2014/03/22. Eng.

14. Rijke YBd. Point-of-Care Creatinine Testing in High-Risk Patients. JIFCC. 2009;20/01.

15. Shephard MDS. Point-of-Care Testing and Creatinine Measurement. The Clinical Biochemist Reviews. 2011;32(2):109-14. PubMed PMID: PMC3100277. 
Tab. 1 Direct interpretation of creatinine results based on the P.I.A. ${ }^{2}$ device.

\begin{tabular}{|c|c|l|}
\hline $\begin{array}{c}\text { Creatinine } \\
\text { [mg/dL] }\end{array}$ & Category & \multicolumn{1}{c|}{ Interpretation } \\
\hline$<20$ & Invalid & $\begin{array}{l}\text { Creatinine concentration is too low. Measurement has } \\
\text { to be repeated at a different time point. }\end{array}$ \\
\hline $20-50$ & Low & $\begin{array}{l}\text { The sample is diluted. Actual drug concentration } \\
\text { might be higher }\end{array}$ \\
\hline $50-150$ & Normal & Creatinine concentration in normal range \\
\hline $150-500$ & High & $\begin{array}{l}\text { Urine is concentrated. Actual drug concentration might } \\
\text { be lower }\end{array}$ \\
\hline$>500$ & Very high & $\begin{array}{l}\text { Very concentrated sample. Actual drug concentration } \\
\text { might be much lower. }\end{array}$ \\
\hline
\end{tabular}


Figure caption:

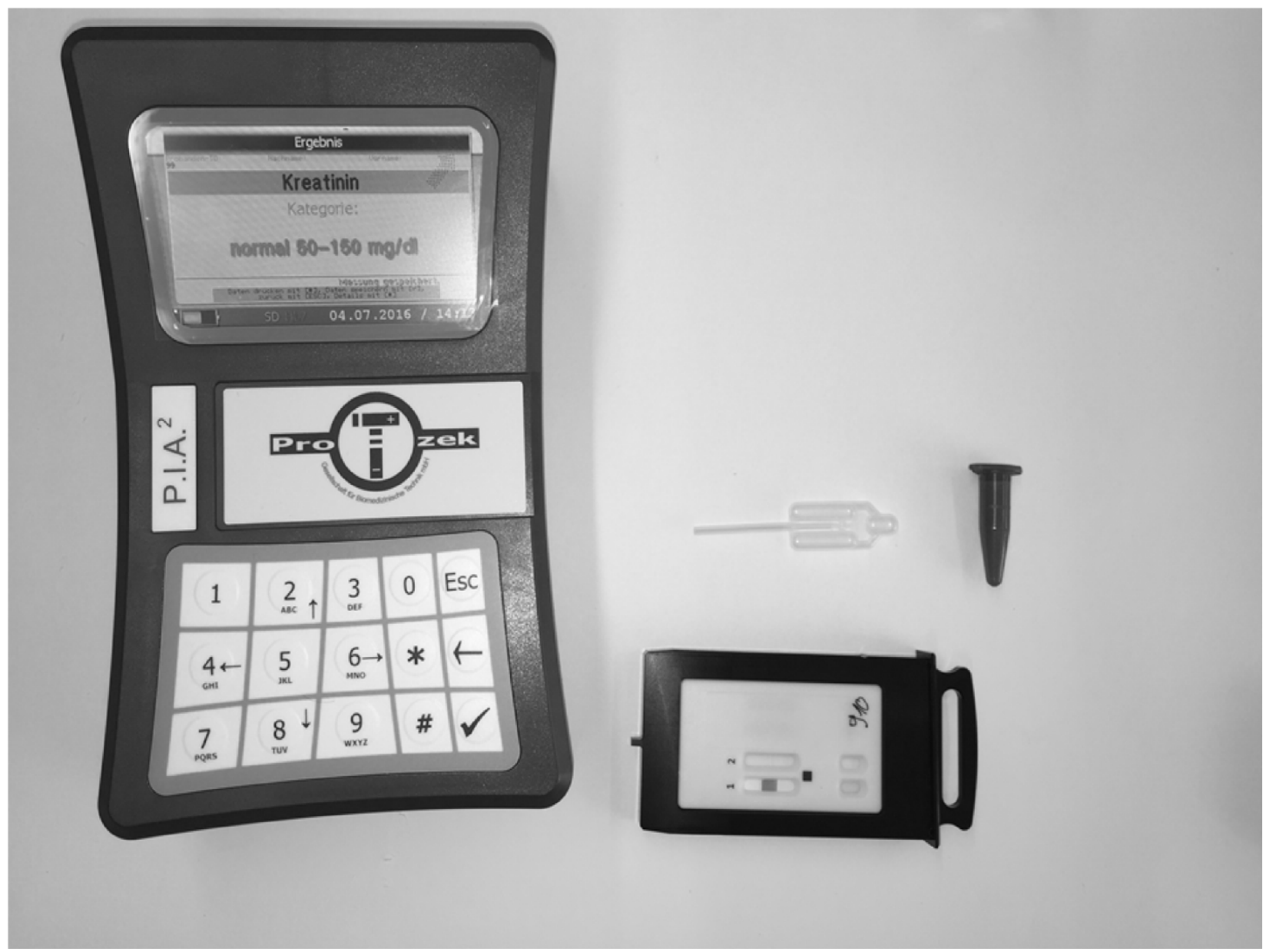

Figure 1 Automatic P.I.A.2 readout system from Protzek with a creatinine test cartridge, a microtube, and a $100 \mu \mathrm{L}$ pipette for the dilution of the urine. 


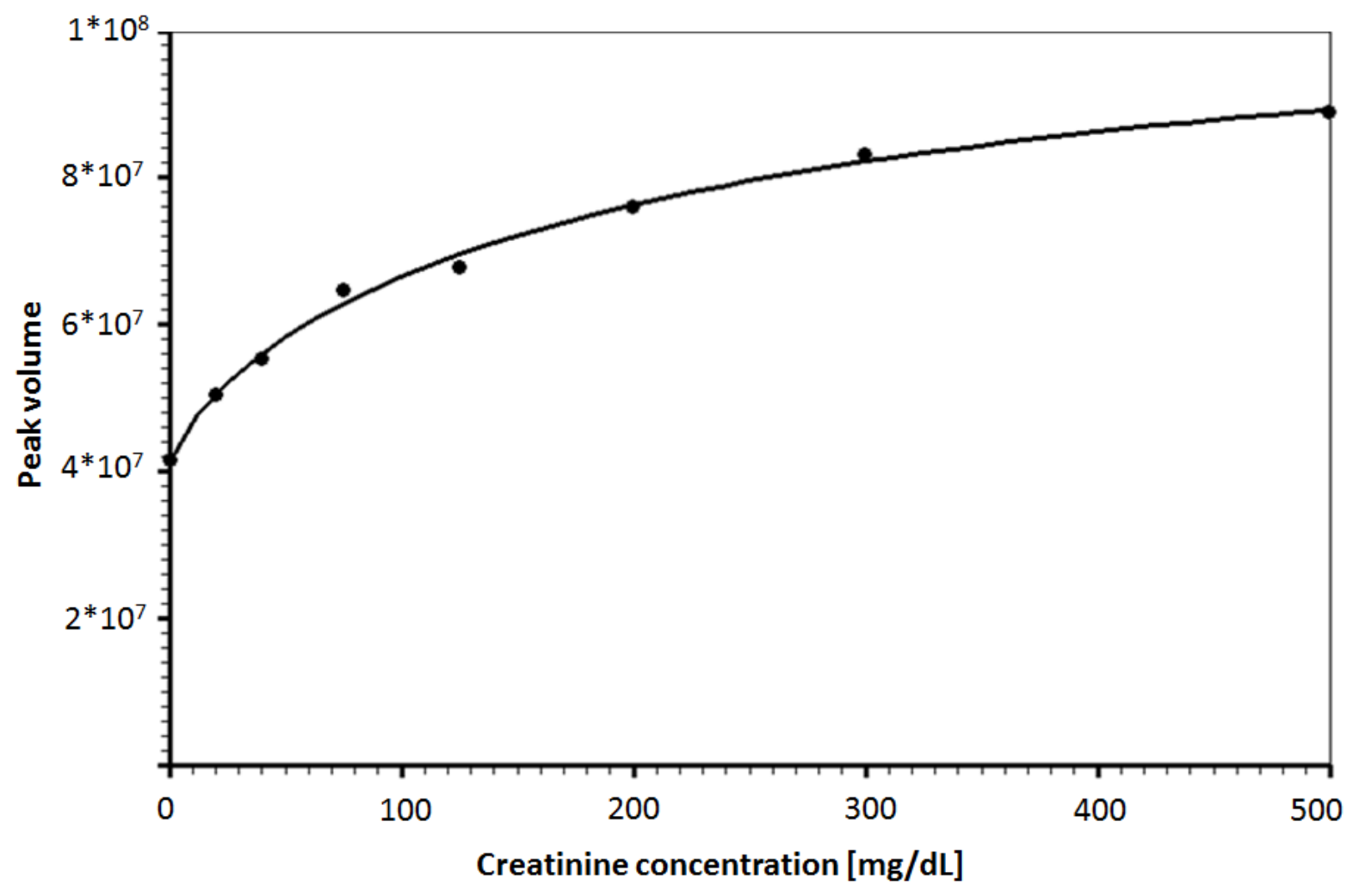

Figure 2 Calibration curve for the POCT device based on a saturation curve established by comparing the peak volume (brightness of the pad) with the spiked creatinine concentration within the sample. (This figure was provided by courtesy of Protzek GmbH) 


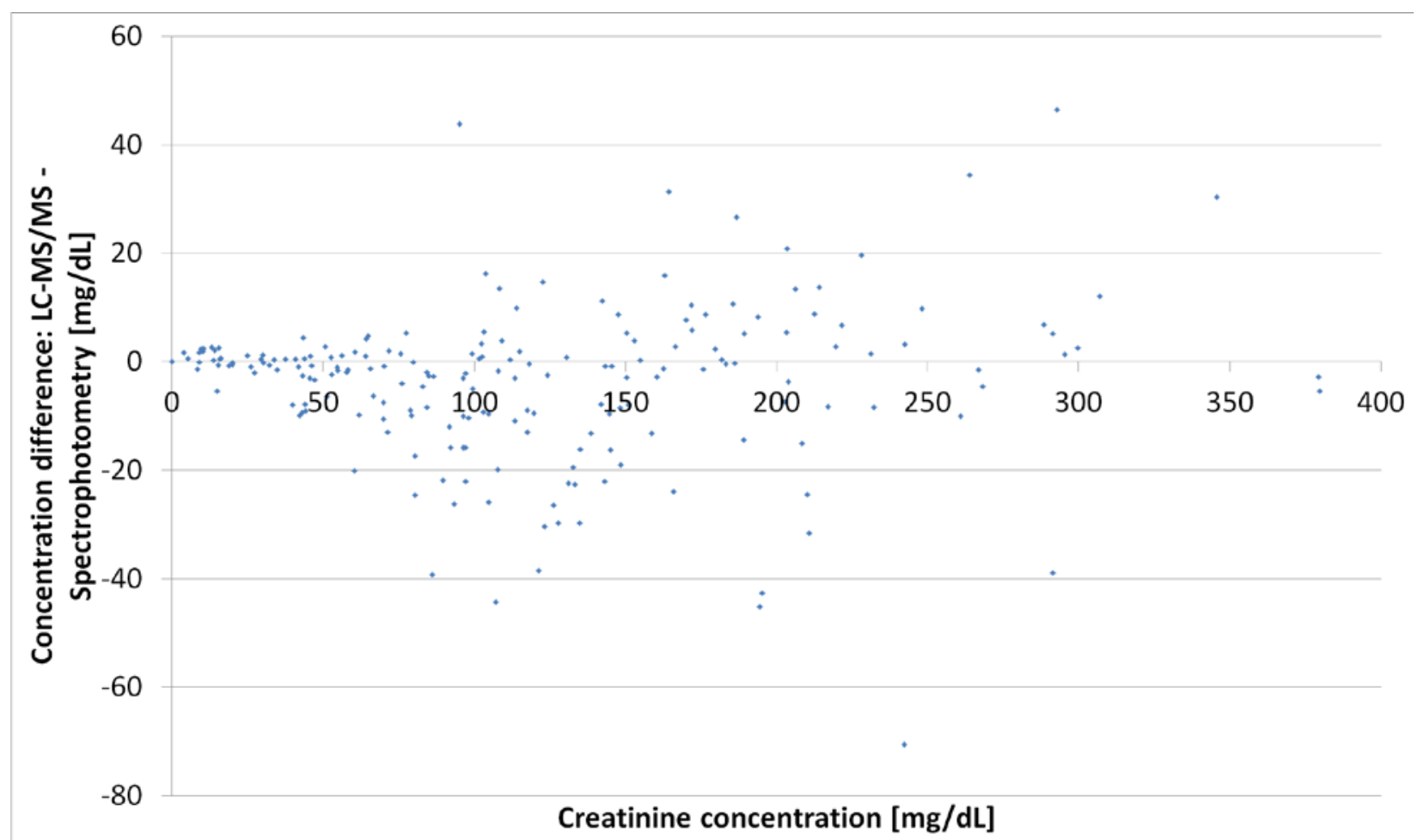

Figure 3 Bland-Altman plot: $\mathrm{S}(\mathrm{x}, \mathrm{y})=((0.5 \times(\mathrm{S} 1+\mathrm{S} 2), \mathrm{S} 1-\mathrm{S} 2))$, whereby $\mathrm{S} 1$ is represented by the LC-MS/MS concentration and S2 by the spectrophotometric AU 480 analyzer concentration.

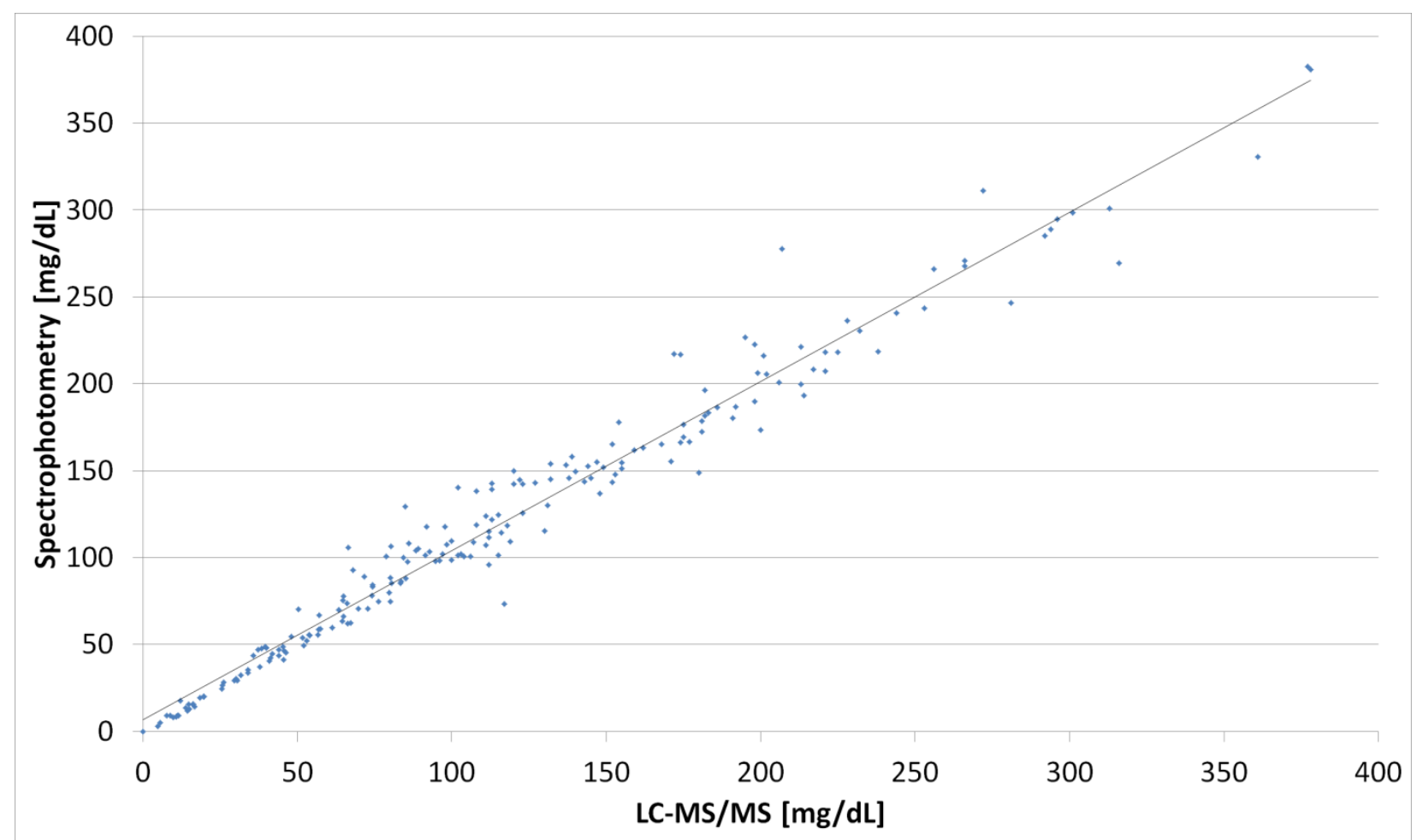

Figure 4 Comparison plot between the spectrophotometric method and the LC-MS/MS method. Linear regression reveals a correlation coefficient $\mathrm{R}^{2}$ of 0.9683 with $\mathrm{y}=0.9732 \mathrm{x}+6.85$ 


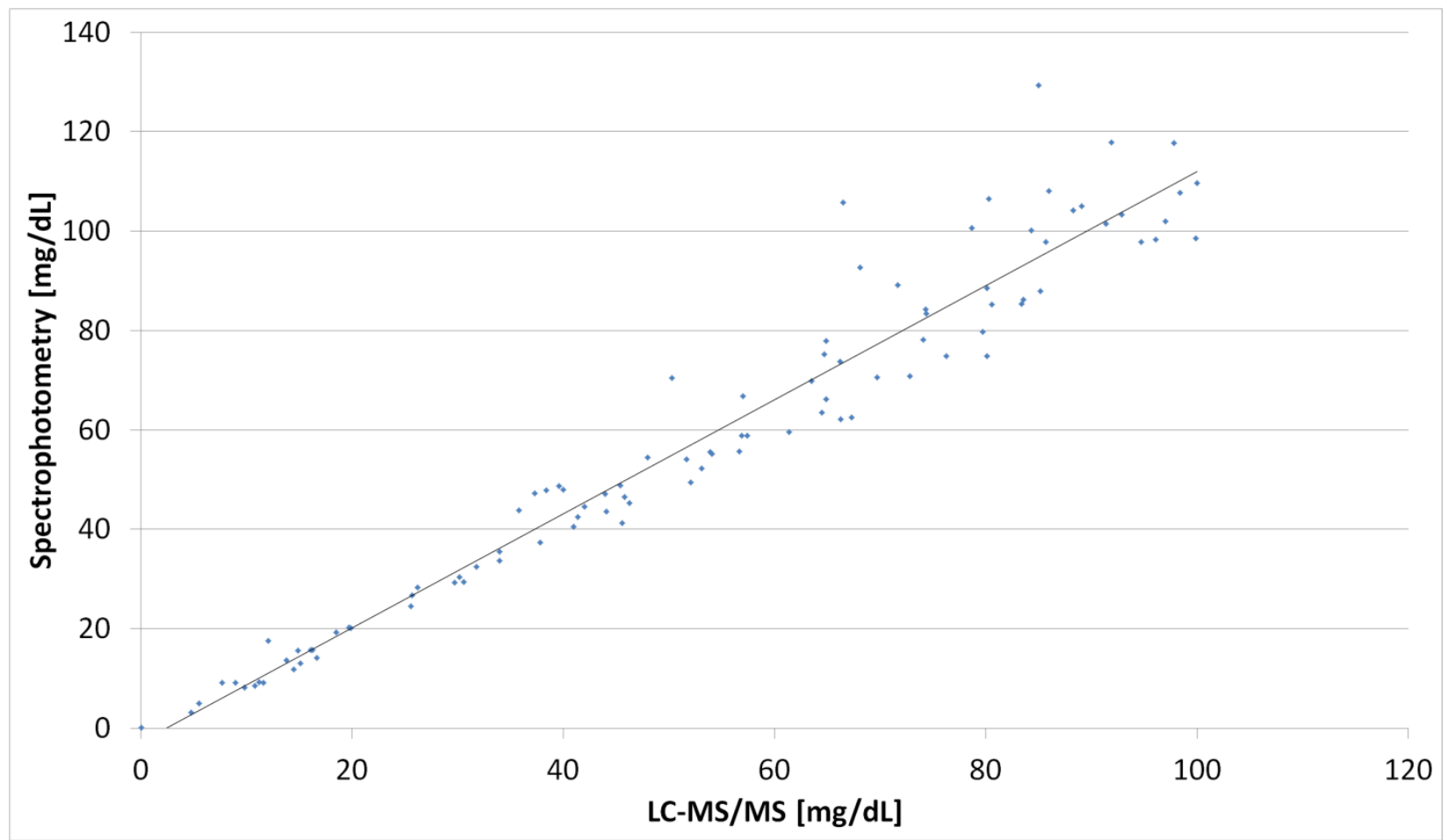

Figure 5 Comparison plot between the spectrophotometric method and the LC-MS/MS method from 0 to $100 \mathrm{mg} / \mathrm{dL}$. Linear regression reveals a correlation coefficient $\mathrm{R}^{2}$ of 0.9435 with $\mathrm{y}=1.1484 \mathrm{x}-2.80$

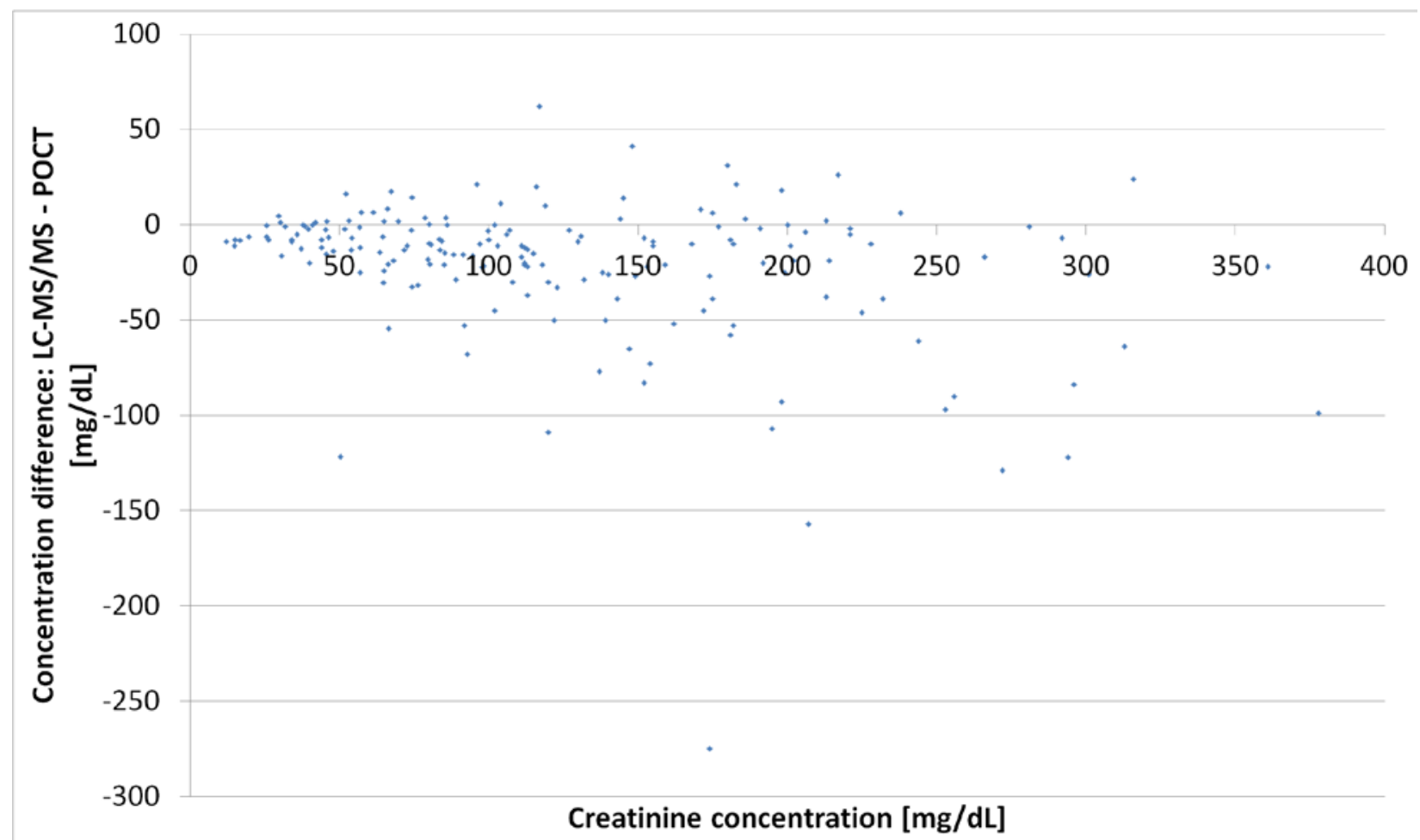

Figure 6 Bland-Altman plot: $\mathrm{S}(\mathrm{x}, \mathrm{y})=((\mathrm{S} 1, \mathrm{~S} 1-\mathrm{S} 2))$, whereby $\mathrm{S} 1$ is represented by the LC-MS/MS 
concentration and S2 by the concentration determined by the POCT device. Samples are presented within the calibration range for POCT (>20 mg/dL (excluding 16 samples) and <500 mg/dL (excluding 4 samples))

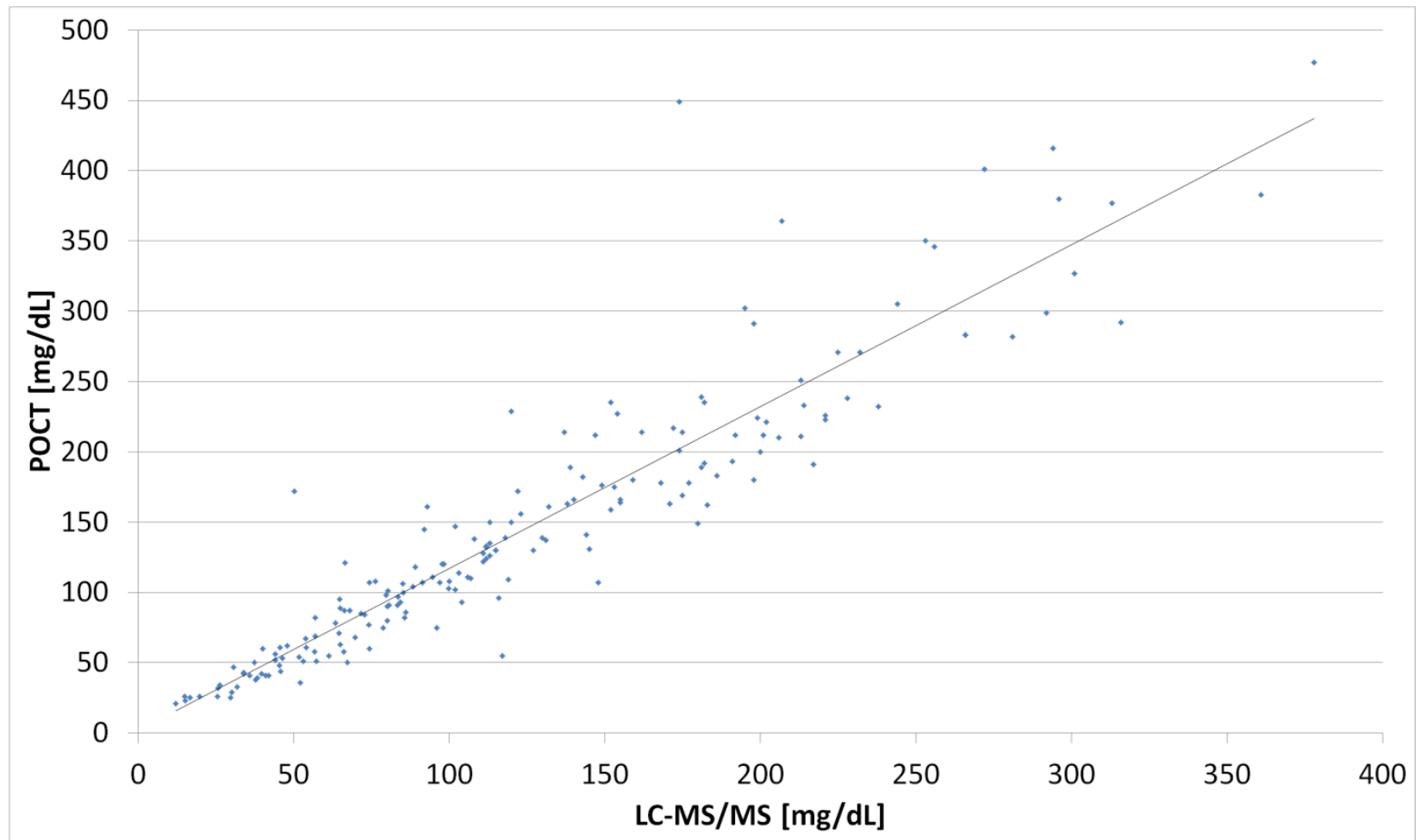

Figure 7 Comparison plot between the POCT device and the LC-MS/MS method. Linear regression reveals a correlation coefficient $\mathrm{R}^{2}$ of 0.8631 with $\mathrm{y}=1.1513 \mathrm{x}+1.80$. Samples are presented within the calibration range for POCT (>20 mg/dL (excluding 16 samples) and <500 mg/dL (excluding 4 samples)) 